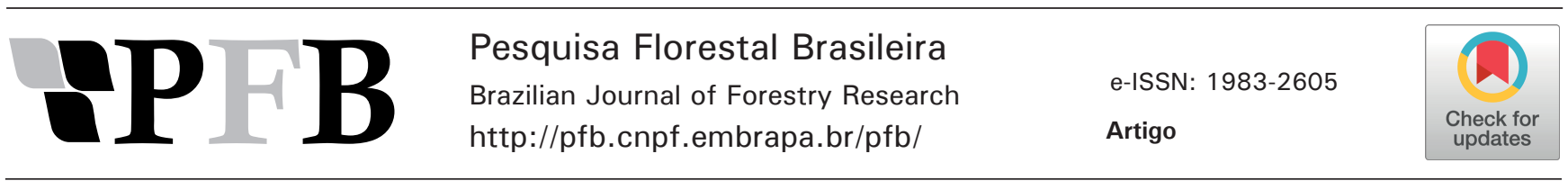

\title{
Distribuição espacial de táxons anemocóricos e zoocóricos em fragmentos de Floresta Ombrófila Mista
}

\author{
Richeliel Albert Rodrigues Silva'(iD)*, Joelmir Augustinho Mazon ${ }^{1}\left[\right.$ iD, Luciano Farinha Watzlawick ${ }^{1}$ (iD \\ ${ }^{1}$ Universidade Estadual do Centro-Oeste, PR 153, Km 7, Riozinho, CEP 84500-000, Irati, PR, Brasil
}

*Autor correspondente:

richeliel@yahoo.com.br

Termos para indexação:

Dispersão de sementes

Araucária

Função K de Ripley

Index terms:

Seed dispersal

Araucaria

Ripley's K Function

Histórico do artigo:

Recebido em 14/08/2018

Aprovado em 01/04/2019

Publicado em 28/06/2019

doi: 10.4336/2019.pfb.39e201801700

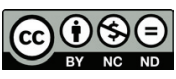

Trabalho apresentado no V Seminário de Atualização Florestal, 24 e 28 de setembro de 2018, Irati, PR.
Resumo - O objetivo desse trabalho foi avaliar o padrão espacial das espécies anemocóricas e zoocóricas em ambientes com diferentes densidades de indivíduos. O estudo foi realizado em dois fragmentos de Floresta Ombrófila Mista Alto-Montana: um sob sistema silvipastoril e outro em uma floresta em processo de sucessão secundária, no município de Turvo, PR. Foram registradas as coordenadas $(\mathrm{x}, \mathrm{y})$ de cada ponto, sendo o padrão espacial analisado com a aplicação da função K de Ripley, na forma univariada, utilizando-se o pacote Splancs. Na área sob sistema silvipastoril, as espécies anemocóricas apresentam-se de forma agregada, enquanto que as espécies com síndrome de dispersão zoocórica apresentam padrão agregado até $15 \mathrm{~m}$ e aleatório nas classes superiores. Na área em sucessão secundária, as espécies anemocóricas estão agregadas em todas as classes de distância. Possivelmente, fatores mais complexos, como a ecologia dos dispersores, relações interespecíficas e características ambientais, podem definir a distribuição espacial de algumas espécies.

\section{Spatial distribution of anemochorics and zoochorics taxons in Araucaria Forest fragments}

\begin{abstract}
The present study had as objective to analyze the spatial pattern of the anemocoric and zoocoric species in environments with different individuals densities. The study was conducted in two fragments of Highland Araucaria Forest: one under silvipastoril system and another in a forest under secondary succession, in the municipality of Turvo, Paraná State, Brazil. The coordinates ( $x, y)$ of each point was registered. The spatial pattern was analyzed through the function K of Ripley, in the univariate form, using the package Splancs. In the area under silvipastoril system, anemochory syndrome is aggregated, while individuals with zoochory dispersion syndrome showed aggregated pattern up to $15 \mathrm{~m}$ and random pattern in the upper classes. Anemocoric species showed aggregated pattern in the area under secondary succession regardless the distance. Probably, more complex factors, such as dispersers' ecology, interspecific relationships and environmental features may define the spatial distribution of some species.
\end{abstract}




\section{Introdução}

Os padrões de distribuição espacial das plantas diferem-se com base em diversos processos e dinâmicas, como competição intra e interespecífica, além de perturbação ou heterogeneidade ambiental e dispersão de sementes - bastante dependentes da fauna e da ocorrência e frequência de ventos (Kang et al., 2017). A ação destes agentes pode determinar os três diferentes padrões de distribuição: agregado, aleatório e uniforme (Townsend et al., 2010).

Vários autores tentam a explicar o funcionamento da dispersão dos propágulos e sua relação com a proximidade da planta-mãe, além da maior competição intraespecífica, atração de predadores e os processos de mortalidade dependentes da densidade. Porém, não parece haver um padrão único para todas as espécies, sendo que alguns modelos se ajustam melhor que outros. Isto acontece porque há outros fatores ecológicos atuando, como filtros e barreiras ambientais, que podem influenciar o padrão de distribuição espacial dos propágulos (Negrini et al., 2012).

Segundo Urbanetz et al. (2003), o padrão espacial assumido por meio da dispersão zoocórica pode se desdobrar em diferentes configurações, dependendo de seu agente dispersor: de aleatório a agregado (morcegos e aves), à uniforme, quando carregados por formigas em seus ninhos. As espécies anemocóricas apresentam hábito frequentemente pioneiro e adaptação a ambientes mais secos e naturalmente menos adensados, conjuntamente com a morfologia, quantidade e peso de suas sementes, tende sempre à aleatoriedade (Budke et al., 2005). Entretanto, algumas espécies anemocóricas podem apresentar padrão diferente, por causa da influência dos fatores antrópicos, como a presença de estradas, pastoreio por bovinos, colheita de produtos não madeireiros ou pela exploração seletiva de indivíduos de grande porte para obtenção de madeira.

A compreensão do padrão de distribuição espacial de uma espécie ou de grupos de espécies é relevante para se compreender a sucessão da floresta e os processos ecológicos, possibilitando com esse conhecimento a definição de estratégias de manejo e conservação da floresta (Rode et al., 2010). Além disso, também é importante para o entendimento dos processos genéticos (Vieira et al., 2010).

A primeira etapa em um estudo sobre a distribuição espacial de espécies de plantas é a descrição do padrão espacial, iniciando-se pela hipótese inicial do padrão espacialmente aleatório, contra duas hipóteses alternativas de padrão agregado e padrão uniforme (Araújo et al., 2014). Partindo deste pressuposto, o objetivo deste trabalho foi verificar se as espécies arbóreas zoocóricas e anemocóricas apresentam distribuição aleatória em remanescentes florestais com diferentes densidades de indivíduos.

\section{Material e métodos}

O estudo foi realizado na propriedade particular Edelweiss, no município de Turvo, localizado na região central do estado do Paraná, entre as coordenadas $446334 \mathrm{E}$ e $7228360 \mathrm{~N}$ (UTM22 J), a uma altitude média de $1.080 \mathrm{~m}$. O clima da região é classificado como Subtropical Úmido Mesotérmico, com verões frescos, sem estações secas e com geadas $(C f b)$, conforme a classificação de Köppen (Alvares et al., 2013), com concentração de chuvas bem distribuídas e estação seca definida, com precipitação anual entre 1.600 e $1.800 \mathrm{~mm}$.

O solo na área de estudo e classificado como Latossolo Bruno Distrófico (Santos et al., 2013), caracterizado pela textura argilosa, álico e relevo suave ondulado, possuindo caráter retrátil e horizonte $\mathrm{A}$ húmico, com conteúdo de carbono orgânico muitas vezes superior a 10 $\mathrm{g} \mathrm{kg}^{-1}$ até $0 \mathrm{~s} 70 \mathrm{~cm}$ de profundidade. Apresenta saturação por bases baixa $(\mathrm{V}<50 \%)$ na maior parte dos primeiros $100 \mathrm{~cm}$ do horizonte $\mathrm{AB}$ e BA, sendo classificado como solo distrófico.

A vegetação natural local é classificada como Floresta Ombrófila Mista de formação Alto-Montana (IBGE, 2012), tendo esta classificação relação estrita com a altitude. A propriedade particular Edelweiss possui 26,4 ha, com histórico de uso do solo em sistema faxinal, desativado há mais de 30 anos. Seu dossel foi raleado, priorizando-se a entrada de luz e o favorecimento de espécies madeireiras e árvores nativas e adensadas de erva-mate (Ilex paraguariensis). O fragmento sob sistema silvipastoril caracteriza-se pela extração de produtos madeiráveis e não madeiráveis e pastoreio de suínos, equinos, caprinos e bovinos. No fragmento de floresta secundária não ocorre, atualmente, qualquer tipo de intervenção antrópica.

\section{Amostragem}

Foram amostrados todos os indivíduos com diâmetro a 1,30 $\mathrm{m}$ acima do solo (DAP) $\geq 5 \mathrm{~cm}$, das famílias com 
síndromes de dispersão anememocórica e zoocórica, por meio de duas parcelas instaladas na propriedade, em áreas com Floresta Ombrófila Mista Alto-Montana, sendo a primeira sob regime silvipastoril, com 2 ha (200 $\mathrm{x} 100 \mathrm{~m}$ ) e, a segunda, em um fragmento florestal isolado fisicamente e em processo de sucessão secundária, com $1,2$ ha $(200 \times 60 \mathrm{~m})$ (Figura 1$)$. Todos os indivíduos foram identificados e georreferenciados, sendo obtidas as coordenadas ( $\mathrm{x}, \mathrm{y})$, com auxílio de fita métrica (Figura 2).

SILVIPASTORIL
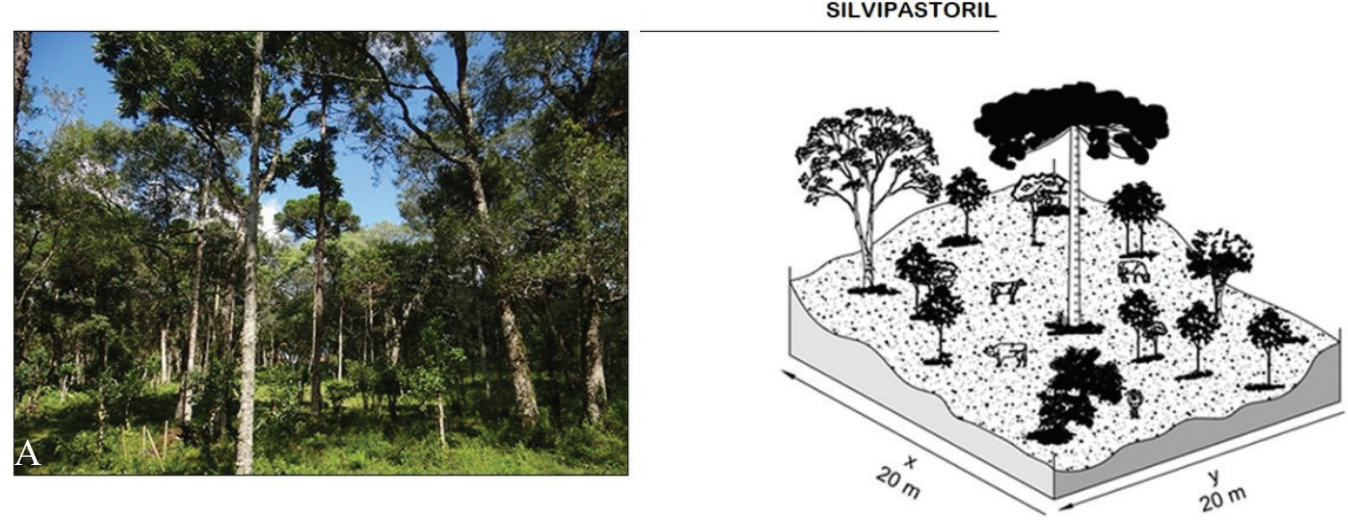

FLORESTA SECUNDÁRIA

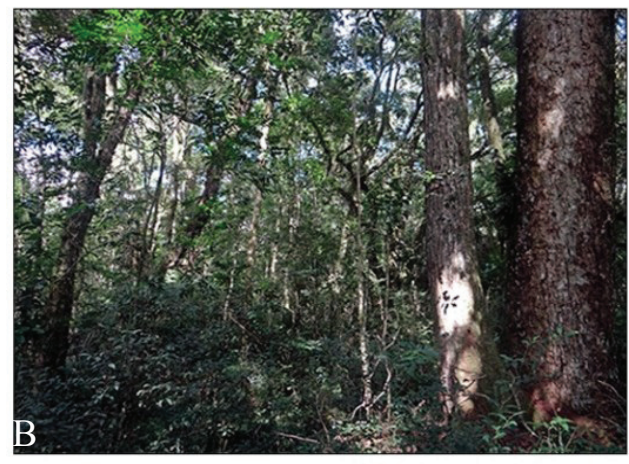

Figura 1. Formações florestais a) em sistema silvipastoril e b) com floresta em processo de sucessão secundária, presentes nas áreas amostradas, Turvo, PR.

Figure 1. Forest formations a) in silvopastoril system and b) with the forest in process of secondary succession, present in the sampled areas, Turvo, Paraná State.

\section{Análises do padrão espacial}

Para análise da distribuição espacial, foi aplicada a função K (Ripley, 1977). Esta função registra todas as distâncias entre as árvores, por meio de um círculo de raio (s), considerando a árvore como o centro, e contabiliza os vizinhos inseridos na área deste círculo.

A hipótese de completa aleatoriedade espacial (CAE) foi testada por meio de intervalos de confiança $99 \%$, a partir de 1.000 simulações. Quando os valores estimados da função $\mathrm{K}$ se encontraram dentro dos intervalos de confiança, for aceita a hipótese nula, ou seja, o padrão foi considerado aleatório. Quando estavam fora dos intervalos de confiança, foi considerada a hipótese alternativa, podendo ocorrer padrão agregado (valores positivos) ou padrão regular (valores negativos).

Para evitar decréscimos nas últimas classes de distância, o padrão espacial foi avaliado até a metade do menor lado da parcela. Diante disso, na área com regime silvipastoril avaliou-se até $50 \mathrm{~m}$, com intervalos de classe de $10 \mathrm{~m}$; e na área em processo de sucessão secundária até $30 \mathrm{~m}$, com intervalos de classe de $5 \mathrm{~m}$. Os valores obtidos pela função $\mathrm{K}$ foram transformados para função L(s), com objetivo de melhorar a compreensão dos valores estimados. As análises foram realizadas no software R 3.5.0 (R Development Core Team, 2008), por meio do pacote Splancs (Rowlingson \& Diggle, 2004). 


\section{Resultados}

Foram amostrados 1.145 indivíduos na área sob sistema silvipastoril, sendo $73 \mathrm{com}$ síndrome de dispersão anemocórica $(6,4 \%)$ e 1.072 zoocórica (93,6\%). Na área de floresta em processo de sucessão secundária foram amostrados 1.596 indivíduos, sendo
380 com síndrome de dispersão anemocórica $(23,8 \%)$ e 1.216 zoocórica $(76,2 \%)$ (Figura 1$)$.

$\mathrm{Na}$ Tabela 1 são apresentadas as síndromes de dispersão das espécies encontradas nas duas áreas de estudo. Do total, 47 espécies (81\%) apresentaram síndrome de dispersão zoocórica e apenas 11 espécies $(19 \%)$ dispersão anemocórica.

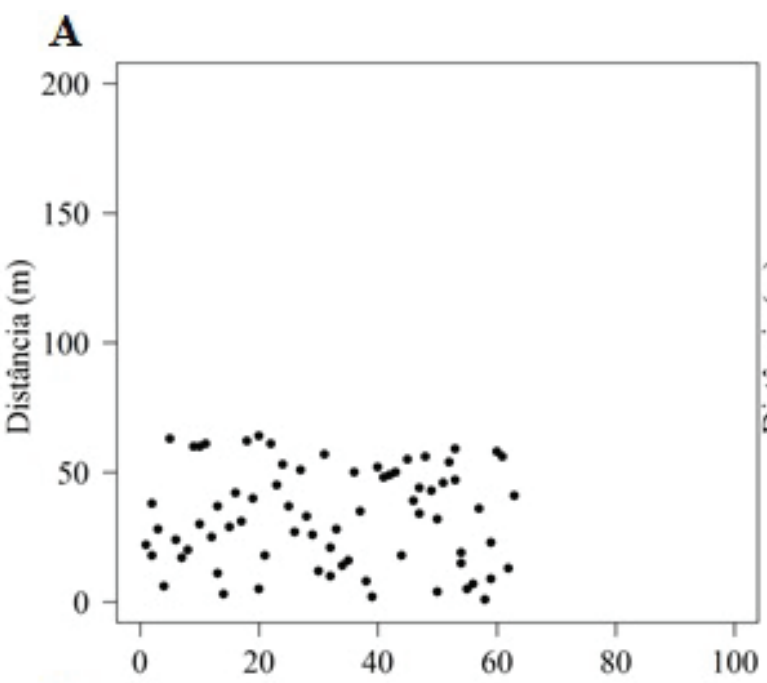

\section{B}
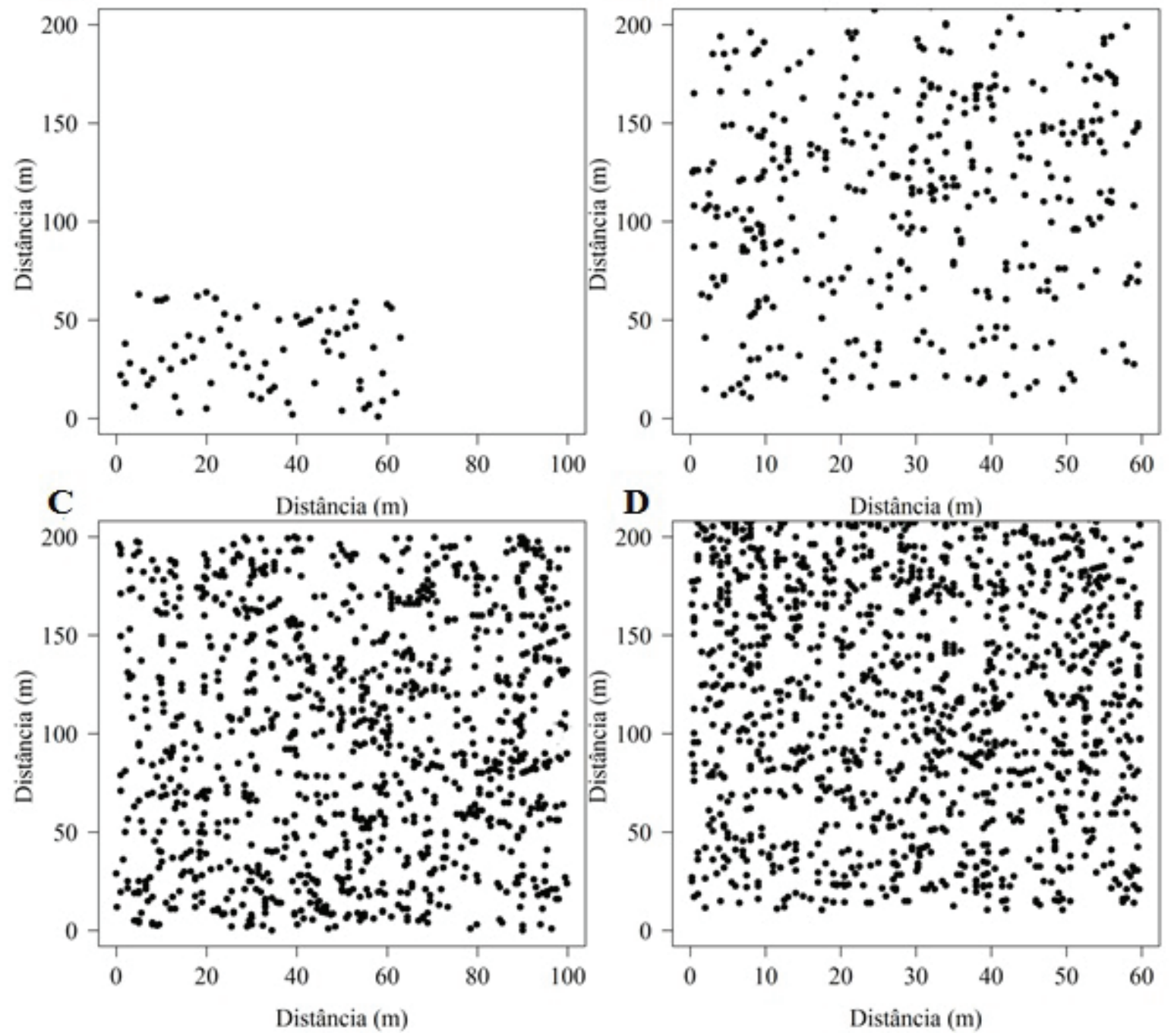

Figura 2. Distribuição espacial dos indivíduos das espécies com síndromes de dispersão anemocórica e zoocórica, Turvo, PR. (A) anemocórica no sistema silvipastoril, (B) anemocórica na floresta secundária, (C) zoocórica no sistema silvipastoril e (D) zoocórica na floresta secundária.

Figure 2. Spatial distribution of individuals of species with anorexic and zoocoric dispersion syndromes, Turvo, Paraná State. (A) anemocoric in the silvipastoril system, (B) anemocoric in the secondary forest, (C) zoocoric in the silvipastoril system and (D) zoocoric in the secondary forest. 
Tabela 1. Espécies registradas nas áreas sob sistema silvipastoril e floresta em sucessão secundária de Floresta Ombrófila Mista Alto-Montana, em Turvo, PR.

Table 1. Species registered in the areas under silvipastoril system and in a forest under secondary succession in Highland Araucaria Forest, in Turvo, Paraná State.

\begin{tabular}{|c|c|c|c|c|}
\hline \multirow{2}{*}{ Família/Espécie } & \multicolumn{2}{|c|}{ Ocorrência } & \multirow{2}{*}{ SD } & \multirow{2}{*}{ Fonte } \\
\hline & SP & FS & & \\
\hline \multicolumn{5}{|l|}{ AQUIFOLIACEAE } \\
\hline Ilex brevicuspis Reissek & - & - & Zoo & Grings \& Brack (2009) \\
\hline Ilex paraguariensis A. St.-Hil. & - & • & Zoo & Carvalho (2003) \\
\hline Ilex theezans Mart. ex Reissek & - & $\bullet$ & Zoo & Carvalho (2008) \\
\hline \multicolumn{5}{|l|}{ ARAUCARIACEAE } \\
\hline Araucaria angustifolia (Bertol.) Kuntze & $\bullet$ & $\bullet$ & Zoo & Carvalho (2010) \\
\hline \multicolumn{5}{|l|}{ ARECACEAE } \\
\hline Syagrus romanzoffiana (Cham.) Glassman & $\bullet$ & $\bullet$ & Zoo & Carvalho (2006) \\
\hline \multicolumn{5}{|l|}{ ASTERACEAE } \\
\hline Dasyphyllum spinescens (Less.) Cabrera & - & $\bullet$ & Ane & Marmontel et al. (2013) \\
\hline Moquiniastrum polymorphum (Less.) G. Sancho & - & $\bullet$ & Ane & Carvalho (2003) \\
\hline Piptocarpha angustifolia Dusén ex Malme & $\bullet$ & $\bullet$ & Ane & Carvalho (2003) \\
\hline Piptocarpha axillaris (Less.) Baker & - & - & Ane & Lima et al. (2011) \\
\hline Vernonanthura discolor (Spreng.) H. Rob. & $\bullet$ & $\bullet$ & Zoo & Carvalho (2008) \\
\hline \multicolumn{5}{|l|}{ BIGNONIACEAE } \\
\hline Jacaranda micrantha Cham. & - & $\bullet$ & Ane & Carvalho (2003) \\
\hline \multicolumn{5}{|l|}{ BORAGINACEAE } \\
\hline Cordia ecalyculata Vell. & - & $\bullet$ & Zoo & Miachir (2009) \\
\hline \multicolumn{5}{|l|}{ CANELLACEAE } \\
\hline Cinnamodendron dinisii Schwanke & - & - & Zoo & Carvalho (2010) \\
\hline \multicolumn{5}{|l|}{ CELASTRACEAE } \\
\hline Maytenus evonymoides Reissek & - & $\bullet$ & Zoo & Catharino et al. (2006) \\
\hline \multicolumn{5}{|l|}{ CLETHRACEAE } \\
\hline Clethra scabra Pers & $\bullet$ & $\bullet$ & Ane & Carvalho (2006) \\
\hline \multicolumn{5}{|l|}{ CUNONIACEAE } \\
\hline Lamanonia ternata Vell. & - & - & Ane & Carvalho (2003) \\
\hline Weinmannia paulliniifolia Pohl ex Ser & $\bullet$ & - & Zoo & Catharino et al. (2006) \\
\hline \multicolumn{5}{|l|}{ CYATHEACEAE } \\
\hline Cyathea corcovadensis (Raddi.) Domin. & - & $\bullet$ & Ane & Marmontel et al. (2013) \\
\hline \multicolumn{5}{|l|}{ DICKSONIACEAE } \\
\hline Dicksonia sellowiana Hook. & $\bullet$ & $\bullet$ & Ane & Grings e Brack (2009) \\
\hline \multicolumn{5}{|l|}{ FABACEAE } \\
\hline Inga vera Willd. & - & - & Zoo & Ferreira et al. (2013) \\
\hline \multicolumn{5}{|l|}{ LAURACEAE } \\
\hline Cinnamomum атоепит (Nees \& Mart.) Kosterm. & • & • & Zoo & Ferreira et al. (2013) \\
\hline Cinnamomum sellowianum (Nees \& Mart.) Kosterm. & - & • & Zoo & Miachir (2009) \\
\hline Nectandra lanceolata Nees & - & • & Zoo & Carvalho (2003) \\
\hline Nectandra megapotamica (Spreng.) Mez & - & - & Zoo & Carvalho (2006) \\
\hline Ocotea diospyrifolia (Meisn.) Mez & • & $\bullet$ & Zoo & Marmontel et al. (2013) \\
\hline Ocotea elegans $\mathrm{Mez}$ & - & $\bullet$ & Zoo & Catharino et al. (2006) \\
\hline Ocotea porosa (Nees \& Mart.) Barroso & $\bullet$ & $\bullet$ & Zoo & Carvalho (2003) \\
\hline Ocotea puberula (Rich.) Nees & - & $\bullet$ & Zoo & Carvalho (2003) \\
\hline Ocotea silvestris Vattimo-Gil & - & $\bullet$ & Zoo & Catharino et al. (2006) \\
\hline
\end{tabular}




\begin{tabular}{|c|c|c|c|c|}
\hline \multirow{2}{*}{ Família/Espécie } & \multicolumn{2}{|c|}{ Ocorrência } & \multirow{2}{*}{ SD } & \multirow{2}{*}{ Fonte } \\
\hline & SP & FS & & \\
\hline \multicolumn{5}{|l|}{ MELASTOMATACEAE } \\
\hline Miconia sellowiana Naudin & - & $\bullet$ & Zoo & Grings \& Brack (2009) \\
\hline \multicolumn{5}{|l|}{ MELIACEAE } \\
\hline Cabralea canjerana (Vell.) Mart & $\bullet$ & - & Zoo & Carvalho (2003) \\
\hline Cedrela fissilis Vell. & - & $\bullet$ & Ane & Carvalho (2003) \\
\hline Trichilia elegans A. Juss. & - & $\bullet$ & Zoo & Dan et al. (2010) \\
\hline \multicolumn{5}{|l|}{ MYRTACEAE } \\
\hline Calyptranthes concinna DC. & • & $\bullet$ & Zoo & Ferreira et al. (2013) \\
\hline Campomanesia xanthocarpa (Mart.) O. Berg & $\bullet$ & $\bullet$ & Zoo & Carvalho (2006) \\
\hline Eugenia hiemalis Cambess. & $\bullet$ & $\bullet$ & Zoo & Callegaro et al. (2012) \\
\hline Eugenia uniflora $\mathrm{L}$. & - & $\bullet$ & Zoo & Ferreira et al. (2013) \\
\hline Eugenia uruguayensis Cambess. & $\bullet$ & - & Zoo & Negrini et al. (2012) \\
\hline Myrcia guianensis (Aubl.) DC. & $\bullet$ & - & Zoo & Ferreira et al. (2013) \\
\hline Myrcia multiflora (Lam.) DC. & • & - & Zoo & Catharino et al. (2006) \\
\hline Myrcia retorta Cambess. & $\bullet$ & $\bullet$ & Zoo & Scherer et al. (2007) \\
\hline Myrcianthes gigantea (D. Legrand) D. Legrand & - & $\bullet$ & Zoo & Callegaro et al. (2012) \\
\hline Myrciaria tenella (DC.) O. Berg & $\bullet$ & $\bullet$ & Zoo & Ferreira et al. (2013) \\
\hline \multicolumn{5}{|l|}{ PRIMULACEAE } \\
\hline Myrsine coriacea (Sw.) R.Br. ex Roem. \& Schult & - & $\bullet$ & Zoo & Carvalho (2003) \\
\hline \multicolumn{5}{|l|}{ RHAMNACEAE } \\
\hline Hovenia dulcis Thunb. & - & $\bullet$ & Zoo & Callegaro et al. (2013) \\
\hline \multicolumn{5}{|l|}{ ROSACEAE } \\
\hline Prunus myrtifolia (L.) Urb. & $\bullet$ & $\bullet$ & Zoo & Carvalho (2003) \\
\hline \multicolumn{5}{|l|}{ RUTACEAE } \\
\hline Zanthoxylum rhoifolium Lam. & $\bullet$ & $\bullet$ & Zoo & Carvalho (2006) \\
\hline \multicolumn{5}{|l|}{ SALICACEAE } \\
\hline Casearia decandra Jacq. & $\bullet$ & $\bullet$ & Zoo & Callegaro et al. (2012) \\
\hline Casearia obliqua Spreng. & $\bullet$ & $\bullet$ & Zoo & Catharino et al. (2006) \\
\hline Casearia sylvestris $\mathrm{Sw}$. & $\bullet$ & $\bullet$ & Zoo & Carvalho (2006) \\
\hline Xylosma ciliatifolia (Clos) Eichler & - & $\bullet$ & Zoo & Ferreira et al. (2013) \\
\hline \multicolumn{5}{|l|}{ SAPINDACEAE } \\
\hline Allophylus edulis (A. St.-Hil. et al.) Hieron. ex Niederl. & - & $\bullet$ & Zoo & Carvalho (2006) \\
\hline Cupania vernalis Cambess & • & $\bullet$ & Zoo & Carvalho (2006) \\
\hline Matayba elaeagnoides Radlk. & $\bullet$ & $\bullet$ & Zoo & Carvalho (2006) \\
\hline \multicolumn{5}{|l|}{ STYRACACEAE } \\
\hline Styrax leprosus Hook. \& Arn. & - & $\bullet$ & Zoo & Carvalho (2008) \\
\hline \multicolumn{5}{|l|}{ SYMPLOCACEAE } \\
\hline Symplocos tenuifolia Brand & $\bullet$ & $\bullet$ & Zoo & Ferreira et al. (2013) \\
\hline \multicolumn{5}{|l|}{ THEACEAE } \\
\hline Laplacea fruticosa (Schrad.) Kobuski & $\bullet$ & $\bullet$ & Ane & Carvalho (2003) \\
\hline \multicolumn{5}{|l|}{ WINTERACEAE } \\
\hline Drimys brasiliensis Miers & $\bullet$ & $\bullet$ & Zoo & Carvalho (2008) \\
\hline
\end{tabular}

Onde: $\mathrm{SP}=$ sistema silvipastoril; FS = floresta secundária; $\bullet=$ presença, - = ausência; $\mathrm{SD}=$ síndrome de dispersão. Zoo = zoocórica; Ane = anemocórica.

Aárea sob sistema silvipastoril apresentou 21 famílias, sendo que a densidade absoluta da comunidade foi de 573 ind.ha ${ }^{-1}$ e área basal 22,5679 $\mathrm{m}^{2}$.ha ${ }^{-1}$. Sete famílias apresentaram síndrome de dispersão anemocórica, com destaque para Dicksoniaceae (44 indivíduos), Cunoniaceae (12 indivíduos) e Meliaceae (10 indivíduos) e daquelas com dispersão zoocórica, destacaram-se Aquifoliaceae (482 indivíduos), Araucariaceae (331 indivíduos) e Lauraceae (118 indivíduos). 
A área de floresta em sucessão secundária apresentou 27 famílias e densidade de árvores 2,3 vezes maior que a área silvipastoril (1.330 ind.ha $\left.{ }^{-1}\right)$ e área basal de $31,94 \mathrm{~m}^{2}$.ha ${ }^{-1}$. Oito famílias apresentaram síndrome de dispersão anemocórica, com destaque para Clethraceae (140 indivíduos), Cyatheraceae (72 indivíduos) e Dicksoniaceae (68 indivíduos) e daquelas com dispersão zoocórica, destacaram-se Primulaceae (206 indivíduos), Aquifoliaceae (181 indivíduos) e Asteraceae (153 indivíduos).

Os padrões de distribuição espacial das espécies com síndromes de dispersão anemocórica e zoocórica, nas áreas do estudo, estão apresentados na Figura 3. Os intervalos de confiança estão identificados pelas linhas pontilhadas e o valor de $\mathrm{K}$ pela linha contínua.

$\mathrm{Na}$ área silvipastoril, as espécies das famílias com síndrome de dispersão anemocórica encontravamse agregadas, por apresentarem valores de $\mathrm{K}$ acima do intervalo de confiança superior. Os indivíduos das espécies com síndrome de dispersão zoocórica apresentaram padrão agregado até $15 \mathrm{~m}$ e aleatório nas classes superiores.

$\mathrm{Na}$ área de floresta em sucessão secundária, as espécies com síndromes zoocórica ou anemocórica apresentaram padrão de distribuição agregada, independente da classe de distância.
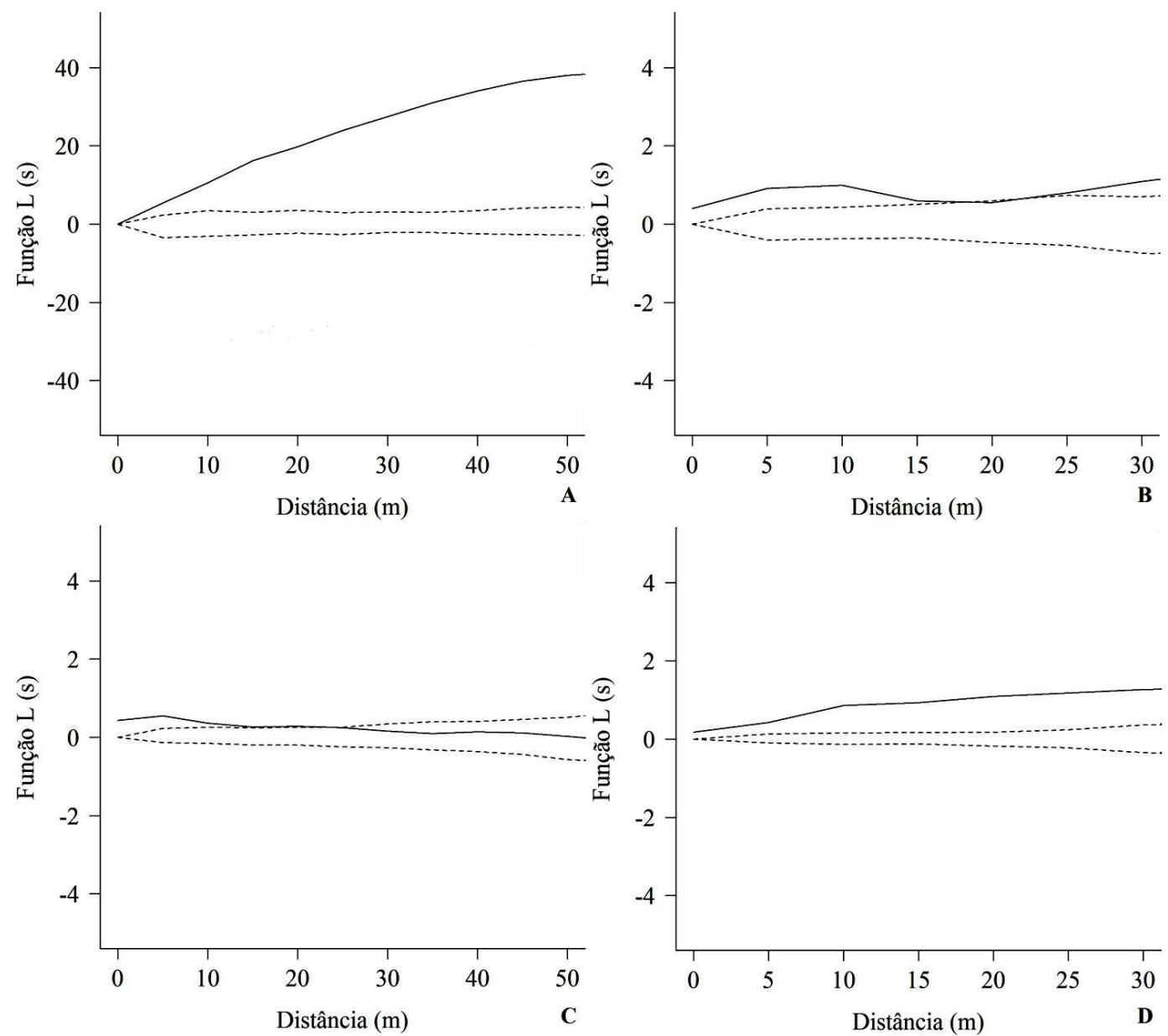

Figura 3. Padrão de distribuição espacial dos indivíduos das espécies com síndromes de dispersão anemocórica e zoocórica, nas áreas sob sistema silvipastoril (SP) e em processo de sucessão secundária (FS), em Turvo, PR. Os intervalos de confiança estão identificados pelas linhas pontilhadas e o valor de K pela linha contínua. A) anemocórica no SP; B) anemocórica na FS; C) zoocórica no SP; e D) zoocórica na FS.

Figure 3. Spatial distribution pattern of individuals of the species with anemocoric and zoocoric dispersion syndromes, in the areas under silvipastoral system (SP) and secondary succession (FS), Turvo, Paraná State. The confidence intervals are identified by dotted lines and the value of $\mathrm{K}$ by the solid line. A) anemocoric in SP; B) anemocoric in FS; C) zoocoric in SP; and D) zoocoric in FS. 


\section{Discussão}

Os padrões de distribuição espacial das espécies ocorrem a partir de diferentes processos, abrangendo interações intra e interespecíficas, considerando a dispersão de sementes próxima ao indivíduo focal. Neste sentido, verificou-se que a hipótese de completa aleatoriedade espacial (hipótese nula) foi aceita apenas para o padrão de distribuição dos indivíduos das espécies zoocóricas no sistema silvipastoril nas maiores classes de distância ( $>25 \mathrm{~m}$ ). Diante disso, diversos fatores podem promover a agregação dos indivíduos, como a predação, disponibilidade de nutrientes, luz, interação com alguma outra espécie ou densidade do estrato inferior da floresta (Paludo et al., 2009).

O padrão bastante agregado das espécies anemocóricas na área silvipastoril aponta que a abertura no dossel, devido ao corte seletivo de indivíduos no passado, influenciou a dispersão. Além disso, as espécies estão zoneadas em uma região de baixa circulação de animais. Neste sentido, estratégias de manejo alteram interações competitivas e propriedades estruturais são afetadas pelas estruturas verticais e horizontais da floresta (Ren et al., 2008).

As espécies com síndrome de dispersão anemocórica apresentam grande densidade de sementes de tamanho pequeno e muitas vezes apresentam estruturas ou alas que permitem a flutuação pelo ar (Van Der Pij1, 1982). Apesar disso, as mesmas não mantiveram tendência à dispersão aleatória nas áreas de estudo (Figura 3), indicando que a dispersão é limitada e que o recrutamento das espécies ocorre próximo à planta-mãe.

As características das espécies na Floresta Ombrófila Mista têm grande influência na sua distribuição, como o peso das sementes e o hábito gregário, principalmente Araucaria angustifolia e Ilex paraguariensis (Silvestre et al., 2012). Associado a isso, os indivíduos estão sujeitos a fatores limitantes ou condicionantes, como a interferência antrópica no ambiente (caso representado pela área silvipastoril), baixa frequência de dispersores e condições edafoclimáticas diferenciadas (Kanieski et al., 2012).

A estratégia de dispersão de espécies com síndrome de dispersão zoocórica é dominante em florestas tropicais, sendo esta característica presente entre $70 \%$ e $95 \%$ das espécies da Mata Atlântica (Peres \& Roosmalen, 2002).
Essa predominância também foi verificada nos dois fragmentos estudados (Tabela 1). A dispersão restrita das sementes nas primeiras classes de distância no sistema silvipastoril (Figura 3) pode ter favorecido o padrão agregado (Vieira et al., 2010).

A maioria das espécies zoocóricas brasileiras possuem bagas carnosas, cujas sementes são potencialmente dispersas por vertebrados frugívoros. No entanto, as espécies apresentadas neste estudo são dispersas majoritariamente por aves (Gressler et al., 2006). Segundo Urbanetz et al. (2003), a tendência natural de sementes dispersas por aves é apresentar o padrão agregado, devido ao hábito de dispersar sementes a partir de um ponto, seja por causa da construção dos ninhos ou do uso destes pontos como poleiros naturais. No entanto, esses mesmos autores mencionam que não há relação entre o mecanismo de dispersão e o padrão espacial, sugerindo que a dispersão de sementes é afetada por fatores mais complexos, como características filogenéticas e adaptações específicas de cada indivíduo. Além disso, uma série de outros fatores, como dispersores secundários e a relação interespecífica após o estabelecimento das plantas, poderia determinar de maneira mais acurada o padrão de distribuição espacial das plantas na regeneração.

\section{Conclusões}

Apesar dos ambientes estudados apresentarem densidades de indivíduos e usos distintos, rejeitouse a hipótese de completa aleatoriedade espacial na distribuição espacial das espécies com síndromes de dispersão zoocórica ou anemocórica.

O padrão de distribuição espacial altamente gregário das espécies anemocóricas na área sob sistema silvipatoril é reflexo das intensas intervenções no passado, por meio da exploração seletiva das espécies de grande porte.

Os resultados mostram diferenças importantes da síndrome de dispersão das espécies, que deveriam ser consideradas na elaboração de programas de conservação, manejo e melhoramento das espécies anemocóricas e zoocóricas. Assim, sugere-se que sejam realizados estudos complementares, como a avaliação da estrutura genética espacial e biologia reprodutiva das espécies. 


\section{Referências}

Alvares, C. A. et al. Köppen's climate classification map for Brazil. Meteorologische Zeitschrift, v. 22, n. 6, p. 711-728, 2013. http:// dx.doi.org/10.1127/0941-2948/2013/0507.

Araújo, E. J. G. et al. Padrão espacial de espécies arbóreas em fragmento de floresta estacional semidecidual. Amazonian Journal of Agricultural and Environmental Sciences, v. 57, n. 2, p. 166171, 2014. http://dx.doi.org/10.4322/rca.2014.010.

Budke, J. C. et al. Composição florística e estratégias de dispersão de espécies lenhosas em uma floresta ribeirinha, arroio Passo das Tropas, Santa Maria, RS, Brasil. Iheringia (Série Botânica), v. 60, n. 1, p. 17-24. 2005.

Callegaro, R. M. et al. Potencial de três plantações florestais homogêneas como facilitadoras da regeneração natural de espécies arbutivo-arbóreas. Scientia Forestalis, v. 41, n. 99, p. 331-341, 2013. http://dx.doi.org/10.14583/2318-7670.v03n04a05.

Callegaro, R. M. et al. Regeneração natural avançada de um fragmento de mata ciliar em Jaguari, RS, Brasil. Revista Brasileira de Ciências Agrárias, v. 7, n. 2, p. 315-321, 2012. http://dx.doi. org/10.5039/agraria.v7i2a1528.

Carvalho, P. E. R. Espécies arbóreas brasileiras. Brasília, DF: Embrapa Informação Tecnológica, 2003. v. 1. 1039 p.

Espécies arbóreas brasileiras. Brasília, DF: Embrapa Informação Tecnológica, 2006. v. 2. 627 p.

Espécies arbóreas brasileiras. Brasília, DF: Embrapa Informação Tecnológica, 2008. v. 3. 593 p.

Espécies arbóreas brasileiras. Brasília, DF: Embrapa Informação Tecnológica, 2010. v. 4. 644 p.

Catharino, E. L. M. et al. Aspectos da composição e diversidade do componente arbóreo das florestas da Reserva Florestal do Morro Grande, Cotia, SP. Biota Neotropica, v. 6, n. 2, p. 1-28, 2006. http:// dx.doi.org/10.1590/S1676-06032006000200004.

Dan, M. et al. Estrutura da comunidade arbórea de fragmentos de floresta estacional semidecidual na bacia hidrográfica do rio São Domingos, Rio de Janeiro, Brasil. Rodriguésia, v. 61, n. 4, p. 749766, 2010. http://dx.doi.org/10.1590/2175-7860201061414.

Ferreira, P. I. et al. Espécies potenciais para recuperação de Áreas de Preservação Permanente no Planalto Catarinense. Floresta e Ambiente, v. 20, 2013. http://dx.doi.org/10.4322/floram.2013.003.

Gressler, E. et al. Pollination and seed dispersal of brazilian Myrtaceae. Brazilian Journal of Botany, v. 29, n. 4, p. 509-530, 2006. http://dx.doi.org/10.1590/S0100-84042006000400002.

Grings, M. \& Brack, P. Árvores na vegetação nativa de Nova Petrópolis, Rio Grande do Sul. Iheringia (Série Botânica), v. 64, n. 1, p. 5-22, 2009.

IBGE. Manual técnico da vegetação brasileira. 2 ed. Rio de Janeiro, RJ, 2012. 217 p.

Kang, H. et al. Population structure and spatial pattern of predominant tree species in a pine-oak mosaic mixed forest in the Qinling Mountains, China. Journal of Plant Interactions, v. 12, n. 1, p. 78-86, 2017. http://dx.doi.org/10.1080/17429145.2017.1283069.
Kanieski, M. R. et al. Diversidade e padrões de distribuição espacial de espécies no estágio de regeneração natural em São Francisco De Paula, RS, Brasil. Revista Floresta, v. 42, n. 3, p. 509-518, 2012. http://dx.doi.org/10.5380/rf.v42i3.25037.

Lima, P. G. C. et al. Plantas medicinais em feiras e mercados públicos do Distrito Florestal Sustentável da BR-163, estado do Pará, Brasil. Acta Botânica Brasilica, v. 25, n. 2, p. 422-434, 2011. http://dx.doi. org/10.1590/S0102-33062011000200018.

Marmontel, C. V. F. et al. Caracterização da vegetação secundária do bioma Mata Atlântica com base em sua posição na paisagem. Bioscience Journal, v. 29, n. 6, p. 2042-2052, 2013.

Miachir, J. Caracterização da vegetação remanescente visando à conservação e restauração florestal no município de Paulínia-SP. 2009. $121 \mathrm{f}$. Tese (Doutorado em Ecologia Aplicada) - Universidade de São Paulo, Piracicaba.

Negrini, M. et al. Dispersão, distribuição espacial e estratificação vertical da comunidade arbórea em um fragmento florestal no Planalto Catarinense. Revista Árvore, v. 36, n. 5, p. 919-929, 2012. http://dx.doi.org/10.1590/S0100-67622012000500014.

Paludo, G. F. et al. Estrutura demográfica e padrão espacial de uma população natural de Araucaria angustifolia (Bertol.) Kuntze (Araucariaceae), na reserva genética florestal de caçador, estado de Santa Catarina. Revista Árvore, v. 33, n. 6, p. 1109-1121, 2009. http://dx.doi.org/10.1590/S0100-67622009000600013.

Peres, C. A. \& Roosmalen, M. V. Patterns of primate frugivory inAmazonia and the Guianan shield: Implications to the demography oflarge-seeded plants in overhunted tropical forests. In: Levey, D. et al. (Ed.). Seed dispersal and frugivory: ecology, evolutionand conservation, Oxford: CABI International, 2002. p. 407-423.

R Development Core Team. R: a language and environment for statistical computing. Vienna: R Foundation for Statistical Computing, 2008.

Ren, H. et al. Nurse plant theory and its application in ecological restoration in lower subtropics of China. Progress in Natural Science, v. 18, n. 2, p. 137-142. 2008. http://dx.doi.org/10.1016/j. pnsc.2007.07.008.

Ripley, B. D. Modelling spatial patterns. Journal of the Royal Statistic Society, v. 39, p. 172-212, 1977.

Rode, R. et al. Análise do padrão espacial de espécies e de grupos florísticos estabelecidos em um povoamento de Araucaria angustifolia em uma Floresta Ombrófila Mista no Centro-Sul do Paraná. Revista Floresta, v. 40, n. 2, p. 255-268, 2010. http://dx.doi. org/10.5380/rf.v40i2.17821.

Rowlingson, B. \& Diggle, P. Splancs: spatial and space-time point pattern analysis. Austria: R Development Core Team, 2004. (R package version 2.01-15).

Santos, H. G. dos et al. Sistema brasileiro de classificação de solos. 3 ed. Brasília, DF: Embrapa, 2013. 353 p.

Scherer, A. et al. Regeneração arbórea num capão de restinga no Rio Grande do Sul, Brasil. Iheringia (Série Botânica), v. 62, n. 1-2, p. 89-98, 2007. 
Silvestre, R. et al. Análise estrutural e distribuição espacial em remanescente de Floresta Ombrófila Mista, Guarapuava (PR). Revista Ambiência, v. 8, n. 2, p. 259-274, 2012. http://dx.doi. org/10.5777/ambiencia.2012.02.03.

Townsend, C. R. et al. (Ed.). Fundamentos em ecologia. 3 ed. Porto Alegre: Artmed, 2010. 576 p.

Urbanetz, C. et al. Padrão espacial, escala e síndromes de dispersão. 2003. Disponível em: <https://www2.ib.unicamp.br/ profs/fsantos/relatorios/ne211r3a2003.pdf>. Acesso em: 23 abr. 2018.
Van Der Pijl, L. Principles of dispersal in higher plants. Berlin: Springer-Verlag, 1982. 162 p.

Vieira, F. A. et al. Spatial pattern and finescale genetic structure indicating recent colonization of the palm Euterpe edulis in a Brazilian Atlantic forest fragment. Biochem Genet, v. 48, p. 96-103, 2010. http://dx.doi.org/10.1007/s10528-009-9298-3. 